\title{
Molecular and Cellular Mechanism of Leukemogenesis of ATL: Emergent Evidence of a Significant Role for $\mathrm{HBZ}$ in HTLV-1-Induced Pathogenesis
}

\author{
Yorifumi Satou ${ }^{1}$ and Masao Matsuoka ${ }^{2}$ \\ ${ }^{1}$ Immunology Section, Division of Infectious Diseases, Imperial College, Wright-Fleming Institute, Norfolk Place, \\ London W2 1PG, UK \\ ${ }^{2}$ Laboratory for Virus control, Institute for Virus Research, Kyoto University, Kyoto 606-8507, Japan
}

Correspondence should be addressed to Yorifumi Satou,y.satou@imperial.ac.uk

Received 1 September 2011; Accepted 17 October 2011

Academic Editor: Charles R. M. Bangham

Copyright ( $) 2012$ Y. Satou and M. Matsuoka. This is an open access article distributed under the Creative Commons Attribution License, which permits unrestricted use, distribution, and reproduction in any medium, provided the original work is properly cited.

Adult T-cell leukemia (ATL) is a leukemia derived from mature CD4 ${ }^{+} \mathrm{T}$ cells and induced by human T-cell leukemia virus type 1 (HTLV-1) infection. Previous studies have revealed many possible molecular and cellular mechanisms of HTLV-1-induced leukemogenesis, but it still remains unknown how HTLV-1 transforms peripheral CD4 T cells in infected individuals. Given the fact that only $2-5 \%$ of infected individuals develop ATL, HTLV-1 infection alone is not sufficient for the transformation of infected cells. Host genetic and epigenetic abnormalities and host immunological status should be considered in attempting to understand the mechanism of the oncogenesis of ATL. Nonetheless, it is obvious that HTLV-1 infection dramatically increases the risk of leukemia generation from peripheral CD4 T-cells, in which the incidence of leukemia is quite low. Furthermore, the evidence that all ATL cases retain the HTLV-1 provirus, especially the $3^{\prime}$ region, indicates that HTLV-1-encoded genes play a critical role in leukemogenesis. Since increasing evidence indicates that the HTLV-1 bZIP factor (HBZ) gene plays a significant role in the pathogenesis of HTLV-1, we will discuss the cellular and molecular mechanism of ATL generation from the virological point of view, particularly focusing on HBZ.

\section{Introduction}

Human T-cell leukemia virus type 1 (HTLV-1) is a complex retrovirus that infects approximately 10 to 20 million people worldwide [1]. In the late 1970s, adult T-cell leukemia (ATL) was identified as a distinct clinical entity based on its clinical and geographical features, suggesting an association with unknown infectious agents [2]. Thereafter, HTLV-1 was identified in a cell line derived from a patient with cutaneous T-cell leukemia in 1980 [3]. HTLV-1 has been shown to immortalize human T-lymphocytes in vitro [4]. In addition, HTLV-1 infection also induces chronic inflammatory diseases, such as HTLV-1-associated myelopathy/tropical spastic paraparesis (HAM/TSP) [5, 6], HTLV-1-associated uveitis [7], and HTLV-1-associated lung diseases [8]. The entire HTLV-1 sequence was determined [9] and various approaches were used to elucidate the pathogenesis of the virus. However, over 30 years after the discovery of HTLV-I, it is still not fully understood how HTLV-1 transforms mature CD4 T cells. Recent studies have provided emerging evidence of the significance of HBZ in HTLV-1 pathogenesis. In this review, we discuss the present understanding of HTLV-I infection from the virological aspect particularly via focusing on the role of the HBZ gene.

\section{The Strategy of Replication in HTLV-1}

Once a retrovirus enters into host cells, the RNA genome is reverse-transcribed into a double-stranded DNA form, which is then integrated into the host chromosomal DNA by the viral integrase. The integrated viral DNA expresses viral genes to produce infectious virions. There are two retroviral replication patterns: de novo infection from infected cells to 
uninfected cells and clonal expansion of infected host cells [10]. Both free viral particle- and cell-to-cell-mediated de novo infection require expression of viral structural proteins and assembly of the viral particle. The expression of Tax enhances the transcription of viral structural genes from the plus strand of HTLV-1 in this situation (Figure 1). HTLV1 has been reported to spread not via free viral particles but via cell-to-cell transmission through the virological synapse [11]. A recent study showed that the biofilm-like extracellular structure of infected cells plays a role in this cell-to-cell transmission of HTLV-1 [12]. This type of viral spread is thought to contribute to initial establishment of a population of infected cells. However the cells expressing Tax could be eliminated by the host CTL (cytotoxic T lymphocyte) response after establishment of host immunity against HTLV-1. After the establishment of anti-HTLV1 immunity, HTLV-1 replicates predominantly using the second form of replication, clonal expansion of infected cells $[13,14]$. In order to escape from the host immunity, HTLV-1 replicates as a provirus by increasing the number of infected host cells. In this phase, survival of HBZ-expressing cells is enhanced by the low immunogenicity of HBZ [15], which could be explained at least in part by the weak binding activity of HBZ peptide to MHC molecules [16].

In the chronic phase of HTLV-1 infection, proviral load becomes stable in most infected individuals; yet there is a broad range of variation of proviral load among infected individuals. Since the variation of HTLV-1 sequence among infected individuals is very limited, host genetic factors including MHC class I molecules are thought to be important determinants of proviral load. Previously Tax has been considered as the most important antigen for the host immune response that controls proviral load [17], but recent evidence concerning lately identified viral protein HBZ has shifted the focus of research. The finding has suggested that individuals who possess MHC alleles which can efficiently bind and present peptides from HBZ have significantly lower proviral load, and are less likely to develop HAM/TSP [16], suggesting that HBZ expression is a critical determinant of viral persistence in chronic phase of HTLV-1 infection. Consistent with this idea, HBZ expression is constitutively detectable whereas Tax expression is frequently suppressed or diminished in ATL cells [18], which could be considered as the most highly expanded clone among many different HTLV-1-infected clones within an infected individual [19]. Even though HBZ might play an important role in viral persistence, in vivo persistence of HTLV-1 is decreased by mutation of other accessory genes, such as $p 12, p 13$, and $p 30$ [20-25], indicating that viral replication and proliferation of infected cells is controlled by these regulatory and accessory genes in harmony.

In summary, previous findings seem to be consistent with the theory that the initial expansion of HTLV-1 infection is due to de novo infection, which is driven by Tax, transactivator of HTLV-1 $5^{\prime}$ LTR. Once the host immune response to HTLV-1 has been established, HTLV-1 propagates in the host mainly by clonal expansion of infected cells via expressing HBZ, a viral antigen with low immunogenicity, as described previously [15].

\section{The Host Cell of HTLV-1}

HTLV-1 has the potential to infect various cell-types such as T cells, B cells, macrophages, and dendritic cells (DCs) [26], but HTLV-1 can induce clonal expansion and transformation almost exclusively in CD4 T cells [10]. The mechanism underlying why HTLV-1 expands and transforms CD4 Tcell population needs to be uncovered. CD4 T-cells are generally partitioned into two subsets, effector $\mathrm{T}$ cells and regulatory $\mathrm{T}$ cells. The former plays crucial role in immune response by secreting cytokines that promote and activate immune systems, whereas the latter has been considered to suppress excessive immune responses to maintain the homeostasis of the immune system. Since the differentiation, function, and homeostasis are quite different between these two T-cell subsets, it is of great importance to consider the characteristics of each subset in order to understand how HTLV-1 utilizes and affects these CD4 T-cell subsets.

3.1. HTLV-1 Infection in Effector CD4 T Cells. To exert the function as effector $\mathrm{T}$ cells, naive $\mathrm{T}$ cells need to encounter their antigens, be activated, and be converted into effector/memory T cells. Previous reports have demonstrated that HTLV-1 infection is more frequently detected in effector/memory CD4 T cells than in naïve CD4 T cells $[27,28]$. There is no compelling evidence to explain this tendency. We would propose three possible explanations as follows.

(i) High Susceptibility to De Novo Infection. De novo infection of HTLV-1 is achieved mainly by cell-to-cell infection, which is initiated by LFA-1-ICAM-1 interaction between infected cell and uninfected cells [29]. Since the expression level of LFA-1 and ICAM-1 in effector/memory CD4 T-cells is higher than that in naïve CD4 T cells [30], effector/memory CD4 $\mathrm{T}$ cells are likely to be more susceptible to de novo cell-to-cell infection than naïve $\mathrm{CD} 4 \mathrm{~T}$ cells.

(ii) High Proliferative Capacity. Effector/memory CD4 T cells proliferate faster than naïve $\mathrm{CD} 4 \mathrm{~T}$ cells in vivo. In vivo labeling of lymphocyte using deuterium-labeled glucose has shown that the doubling time of effector/memory CD4 T cells is 28 days, which is much shorter than the doubling time of naïve CD4 T cells, 199 days [31]. Long-term survival, a hallmark of memory CD4 $\mathrm{T}$ cell clones, also could contribute to the maintenance of HTLV-1 in vivo. Therefore HTLV1 infection in effector/memory CD4 T-cells is beneficial to clonal expansion of infected cells. Furthermore, HTLV1-infected effector/memory CD4 $\mathrm{T}$ cells are reported to proliferate significantly faster than uninfected cells in vivo in HTLV-1 infected individuals [32].

(iii) Enhancement of the Differentiation from Naïve to Effector/Memory CD4 T Cells. Little is known about the impact of HTLV-1 infection on CD4 T-cell differentiation, because few studies have been focused on the effect of viral gene expression on T-cell differentiation to date. We have recently reported that the proportion of effector/memory CD4 T cells was increased in HBZ-transgenic (HBZ-Tg) mice [33], 


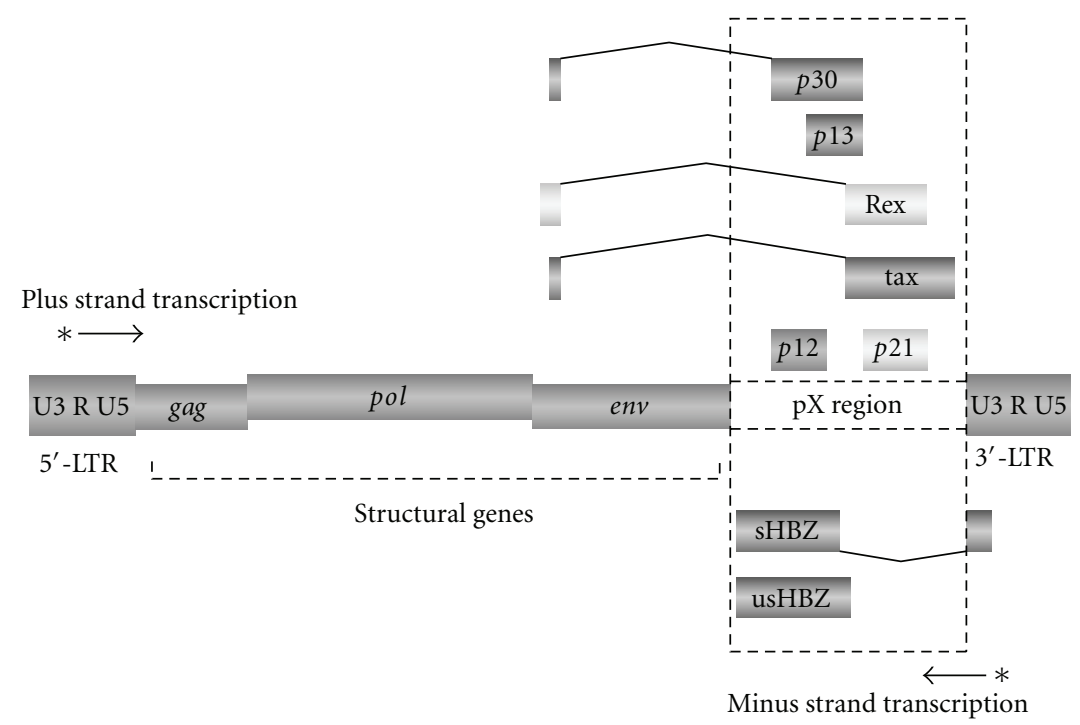

Figure 1: The structure of HTLV-1. HTLV-1 encodes accessory and regulatory genes in the pX region as well as viral structural genes.

suggesting that $\mathrm{HBZ}$ expression can drive the differentiation from naïve $\mathrm{T}$ cells to effector $\mathrm{T}$ cells or enhance cell proliferation more strongly in effector/memory $\mathrm{T}$ cells than naïve $\mathrm{T}$ cells.

HTLV-1 utilizes this effector CD4 T-cell population as a host cell. It follows that this may induce the dysregulation of helper and effector function, contributing to the viral persistence.

3.2. HTLV-1 Infection of Regulatory CD4 $T$ Cells. $\mathrm{CD} 4{ }^{+} \mathrm{CD} 25^{+} \mathrm{FoxP}^{+}$regulatory $\mathrm{T}$ cells (Tregs) have been identified as one of the major immunoregulatory mechanisms which prevent autoimmune disease [34]. Tregs are also involved in the downregulation of specific immune responses during infectious diseases. It has been reported that the frequency of Tregs is elevated in chronic viral infection, such as hepatitis C virus (HCV) [35]. The increased frequency of Tregs may help to prevent immune pathology but on the other hand may facilitate viral persistence. Indeed, the frequency of $\mathrm{CD}^{+} \mathrm{FoxP}^{+} \mathrm{Tax}^{-}$ T cells is inversely correlated with HTLV-1 specific CTL response, which could explain the variation of CTL response among infected people [36]. In addition to this general role of Tregs in chronic viral infection, there is a unique role of Tregs in HTLV-1 infection due to direct infection of CD4 $\mathrm{T}$ cells by HTLV-1, which include both progenitor Treg cells and Treg cells. The frequency of HTLV-1 infection in $\mathrm{CD}^{+}{ }^{+} \mathrm{FoxP}^{+}$cells is higher than other T-cell population [36], which we suggest maybe due to the following.

(i) High Susceptibility to De Novo Infection. Treg cells are known to contact with DCs frequently [37], which could increase the chance of de novo infection between DCs and Tregs. DCs are susceptible to HTLV-1 infection, and HTLV1 infected DCs stimulate proliferation of $\mathrm{T}$ cells $[38,39]$. A recent study also demonstrated that cell-free HTLV-1 efficiently infects DCs, and the infected DCs promote de novo infection of CD4 T cells [40].

(ii) High Proliferative Capacity. In vivo labeling of lymphocytes using deuterium-labeled glucose has shown that the $\mathrm{FoxP}^{+}$Tregs were extremely proliferative in vivo with a doubling time of 8 days [41]. HTLV-1 infection could further enhance proliferative activity of Tregs via expression of HBZ, which has been shown in Tregs of HBZ-Tg mice [33].

(iii) Enhancement of the Differentiation of Tregs by HTLV-1 Infection. $\mathrm{HBZ}$ enhances the generation of $\mathrm{CD} 4^{+} \mathrm{FoxP}^{+} \mathrm{T}$ cells in transgenic mice, suggesting that HBZ has enhancing effect on generation and/or expansion of Foxp $3^{+}$Treg cells. As a mechanism, HBZ promotes the generation of FoxP3 ${ }^{+}$ Tregs via enhancing the TGF- $\beta$ signaling pathways [42], which is a crucial pathway for generation of induced Tregs.

(iv) Advantage of Escape from the Host Immune Systems. Tregs have an immune suppressive effect through both cell-contact-dependent and independent mechanisms [34]. Thus HTLV-1-infected Tregs should be more resistant to HTLV-1-specific CTL killing than HTLV-1-infected nonTregs, resulting in preferential survival of HTLV-1-infected Tregs in vivo.

It seems reasonable that ATL cells would be stochastically derived from FoxP3 ${ }^{+}$Tregs because of the high frequency of HTLV-1 infection in FoxP3 ${ }^{+}$Tregs. But it still remains unclear whether or not ATL is leukemia of FoxP3 ${ }^{+}$Tregs. Some studies have reported that ATL cells have regulatory function $[43,44]$, whereas other studies reported no regulatory function in ATL $[45,46]$. Given the fact that the detection of FoxP3 expression in ATL cells is variable [47, 48], ATL is likely to be derived from both Treg cells and non-Treg cells. But the situation is more complicated because recent studies have indicated there is a plasticity between 
Treg cells and non-Treg cells [49]. Even when ATL cells do not express FoxP3, we cannot exclude the possibility that ATL cells were derived from FoxP $3^{+}$Tregs which have lost the FoxP3 expression during the process of leukemogenesis. Conversely, even when ATL cells do express FoxP3, we cannot exclude the possibility that FoxP3 expression is aberrantly induced in non-Tregs by HTLV-1 infection. Furthermore, even when regulatory function of FoxP3 ${ }^{+}$ATL cells is not evident, there is a possibility that HBZ expression inhibits the function of FoxP3 and impairs the function of the host Tregs [33].

Taken together it is difficult to make a clear conclusion about the relationship between HTLV-1 and the CD4 Tcell subset of host cells at present, but we need to continue the effort to reconcile the complexity to understand the pathogenesis of HTLV-1.

\section{The Minus Strand Viral Gene: HBZ}

The presence of a transcript from the minus strand of the HTLV-1 provirus has been reported in 1989 [50], but HBZ has not been described until recently. HBZ was identified using the CREB-2 binding protein in the yeast two-hybrid screening system using HTLV-1 infected MT-2 cells [51]. HBZ suppresses Tax-mediated viral gene expression from the $5^{\prime}$ LTR by interacting with CREB-2. Therefore, HBZ is a negative regulator for viral gene expression. The following data describing expression of HBZ in primary HTLV-1infected cells have highlighted the significant role of $\mathrm{HBZ}$ in the pathogenesis of HTLV-1 [18, 52-54].

\subsection{Minus Strand Transcription of HTLV-1}

4.1.1. Transcriptional Pattern of HBZ. There are two major transcripts of HBZ, spliced and unspliced HBZ (Figure 1) $[18,55,56]$. Both HBZ transcripts have been detected in ATL cells [56]. Spliced HBZ mRNA expression is correlated with disease severity of HAM/TSP [53]. The level of HBZ expression is higher than that of tax in uncultured primary cells $[52,53]$. A recent study has proposed one possible reason why immunogenicity of HBZ is low. They reported that HBZ-RNA is preferentially retained in the nucleus, which may result in the low translation efficiency of $\mathrm{HBZ}$ and contribute to its immune escape [57]. It is of great interest to elucidate the mechanism why HBZ-RNA is retained in the nucleus because that also could also explain how HBZ RNA induces a growth-promoting effect on T cells [18].

\subsubsection{Possible Effect of Integration Site on HBZ Transcription.} It is possible to evaluate the expression level of HBZ in total PBMC at population level but not at single clone level at present. There are many different clones even within one infected individual [19]. Every infected clone has its own unique integration site; so the difference in the integration site may affect the expression of HBZ; for example, the epigenetic features of HTLV-1 provirus are determined in part by surrounding host genomic features. When HTLV-1 integrated in heterochromatic region of the host genome,
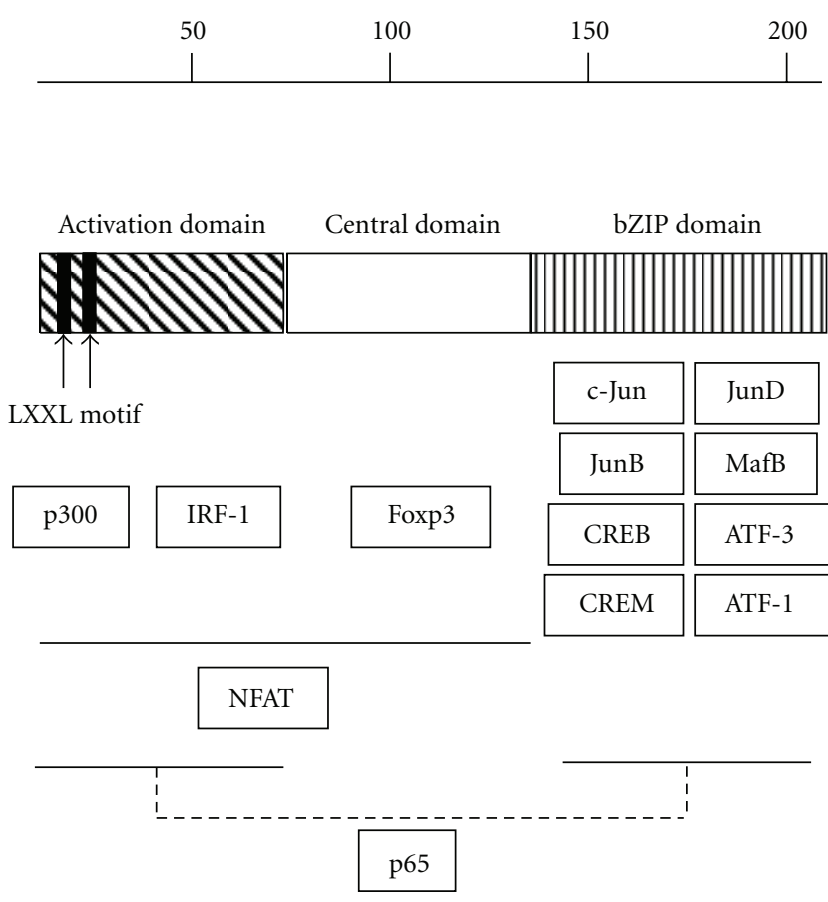

FIgURE 2: The structure of $\mathrm{HBZ}$ and interacting host factors. HBZ could play a crucial role in the HTLV-1 pathogenesis by interacting cellular factor as shown in this figure.

the transcriptional machinery has difficulty accessing the 3'LTR promoter region of HBZ (Figure 1) [58], which could inhibit transcription of HBZ. A previous study has indeed shown that integration sites in ATL cells are frequently located within transcriptional units of host genes but rarely located in a heterochromatic region of human genome compared with those in untransformed infected cells [59]. This result suggests that an HTLV-1-infected clone that carries a provirus integrated within a transcriptionally active region is susceptible to ATL generation. A novel powerful method of integration site analysis using a high-throughput technique has been developed recently and provided more detailed and precise information on the integration site of HTLV-1 [19]. In order to elucidate the effect of the integration site on the clonal dominance, further experiments will be required to investigate the underlying molecular mechanism of the transcriptional interaction between $H T L V-1$ provirus and the surrounding host genome.

4.2. Molecular Property of HBZ Protein. HBZ has been reported to interact with various host factors via its three distinct coiled-coil domains, including activation domain, central domain, and bZIP domain (Figure 2). Transcriptional coactivator p300 and interferon regulatory factor IRF-1 interact with the activation domain of $\mathrm{HBZ}[60,61]$. HBZ induces the activation of TGF- $\beta$ signaling pathway by forming a complex with p300 and Smad proteins [42]. HBZ also activates Dkk1 expression through its KIX domain that interacts with p300 $[60,62]$. The central domain of HBZ is responsible for the interaction with Foxp3 [33], which results 
in the dysfunction of transcriptional activity of Foxp3. The bZIP domain of HBZ is responsible for the interaction with the host bZIP factors, such as c-Jun, JunB, JunD $[63,64]$, CREB, CREM, ATF-1 [65], ATF-3 [66], and MafB [67]. These data have demonstrated that HBZ can form complexes with host factors, resulting in the dysregulation of the host cellsignaling pathways. Although it is very difficult to identify how each molecular mechanism affects the fate of infected cells, it could contribute to the phenotype of infected cells in a coordinated manner. It is striking that HBZ interacts with transcription factors that play a critical role in CD4 $\mathrm{T}$ cells such as AP-1, NF- $\kappa \mathrm{B}$ [68], and FoxP3; therefore these molecular interactions could explain the observed cell-type specificity of transformation induced by HTLV-1.

\subsection{Effect of HBZ Expression on Viral Persistence and HTLV-} 1-Related Pathogenesis. Experiments using a molecular clone of HTLV-1 deleted for HBZ demonstrate that HBZ is dispensable for the HTLV-1-mediated T-cell transformation in vitro. However HBZ plays an indispensable role in persistent viral infection in vivo [69]. More recently, in the macaque model of HTLV-1 infection, reversion of HBZ knock-out HTLV-1 to wild-type HTLV-1 was observed within weeks from infection, also indicating that HBZ plays a crucial role in the persistent infection of HTLV-1 [24]. Tax is thought to be responsible for the in vitro transformation induced by HTLV-1 infection, in which Tax is allowed to express because of the absence of selection pressure by the host immune systems. Also, since Tax has a strong capability to induce genomic instability in infected cells [70-73], Tax could contribute to the accumulation of host genomic abnormalities related to oncogenesis, even at its limited expression level in vivo. Nonetheless, Tax-expressing cells would be susceptible to elimination by CTL in an immune competent host. In contrast, the immune selective pressure on HBZ may be significantly lower allowing expression of HBZ mRNA in vivo $[15,16]$, which should contribute to the persistence of HTLV-1. More surprisingly, the phenotype of HBZ-Tg mice has demonstrated that HBZ expression in CD4 T cells in vivo could induce the disease phenotype of HTLV-1 infection such as chronic inflammation and T-cell lymphoma [33]. Thus, the transgenic expression of either Tax or HBZ induces both T-cell lymphoma and chronic inflammation [33, 7476]. It is of great interest how Tax and HBZ synergistically, competitively, or independently contribute to various aspects of the viral pathogenesis. For example, Tax activates the $\mathrm{NF}-\kappa \mathrm{B}$ signaling pathway, but $\mathrm{HBZ}$ represses the canonical pathway of NF- $\kappa$ B [42]. A recent study has shown that HBZ can alleviate cellular senescence induced by Tax-mediated NF- $\kappa$ B hyper-activation [77]. Future experiments should aim to clarify the role of Tax and HBZ in each aspect of HTLV-1associated pathogenesis.

\section{Future Direction of Treatment}

Since the identification of ATL as a distinct clinical entity, some progress has been made in preventing and treating the disease. In particular, the identification of a transmission route from the mother to her child through breast milk enables us to reduce de novo HTLV-1 infection [78]. However, an effective therapeutic strategy for ATL remains elusive. In particular, there are few available therapies that target HTLV-1 to date. Some reports have shown the efficiency of combination therapy of zidovudine and interferon alpha (AZT/IFN) for treatment of ATL [79], even though little is known about underlying mechanism of AZT/IFN therapy. Recent approaches using allogeneic bone marrow transplantation have significantly improved the prognosis of ATL patients $[80,81]$, suggesting that enhancement of the immune response to HTLV-1 is a possible strategy for treatment of HTLV-1-associated human diseases [82]. As we discussed in this review, if we could activate viral gene expression, we could remove the infected cells by recruiting HTLV-1-specific CTLs. Several preclinical and clinical studies using valproate, a histone deacetylase (HDAC) inhibitor, have already been performed and shown the efficacy of this therapeutic approach. Histone modification is reported to contribute to the proviral gene expression of HTLV-1 [8386]. Treatment with valproate enhances the expression of Tax, and the resulting exposure to anti-Tax CTL may explain the observed reduction in HTLV-1 proviral load [87, 88]. The combination of HDAC inhibitor and antiretroviral drugs remarkably reduced the proviral load in STLV-1 naturally infected baboons [89]. In order to find more efficient and specific molecular therapeutic targets, it is necessary for us to clarify the nature of leukemogenesis induced by HTLV-1.

\section{Acknowledgments}

The authors thank Dr. Aileen Rowan and Dr. Lucy Cook for helpful discussion and critical reading of the manuscript. This work was supported by a grant-in-aid for scientific research from the Ministry of Education, Science, Sports and Culture of Japan, a grant from Naito Foundation, and a grant from Takeda Science Foundation. Y. Satou is supported in part by the fellowship of Yasuda Medical Foundation, Kyoto University Foundation, and Astellas Foundation for Research on Metabolic Disorders.

\section{References}

[1] F. A. Proietti, A. B. F. Carneiro-Proietti, B. C. Catalan-Soares, and E. L. Murphy, "Global epidemiology of HTLV-I infection and associated diseases," Oncogene, vol. 24, no. 39, pp. 60586068, 2005.

[2] T. Uchiyama, J. Yodoi, and K. Sagawa, "Adult T-cell leukemia: clinical and hematologic features of 16 cases," Blood, vol. 50, no. 3, pp. 481-492, 1977.

[3] B. J. Poiesz, F. W. Ruscetti, and A. F. Gazdar, "Detection and isolation of type $\mathrm{C}$ retrovirus particles from fresh and cultured lymphocytes of a patient with cutaneous T-cell lymphoma," Proceedings of the National Academy of Sciences of the United States of America, vol. 77, no. 12, pp. 7415-7419, 1980.

[4] I. Miyoshi, I. Kubonishi, and S. Yoshimoto, "Type C virus particles in a cord T-cell line derived by co-cultivating normal human cord leukocytes and human leukaemic T cells," Nature, vol. 294, no. 5843, pp. 770-771, 1981. 
[5] M. Osame, K. Usuku, and S. Izumo, "HTLV-I associated myelopathy, a new clinical entity," Lancet, vol. 1, no. 8488, pp. 1031-1032, 1986.

[6] A. Gessain, F. Barin, and J. C. Vernant, "Antibodies to human T-lymphotropic virus type-I in patients with tropical spastic paraparesis," Lancet, vol. 2, no. 8452, pp. 407-410, 1985.

[7] M. Mochizuki, K. Yamaguchi, K. Takatsuki, T. Watanabe, S. Mori, and K. Tajima, "HTLV-I and uveitis," Lancet, vol. 339, no. 8801, p. $1110,1992$.

[8] M. Sugimoto, H. Nakashima, S. Watanabe et al., "T-lymphocyte alveolitis in HTLV-I-associated myelopathy," Lancet, vol. 2, no. 8569, p. 1220, 1987.

[9] M. Seiki, S. Hattori, Y. Hirayama, and M. Yoshida, "Human adult T-cell leukemia virus: complete nucleotide sequence of the provirus genome integrated in leukemia cell DNA," Proceedings of the National Academy of Sciences of the United States of America, vol. 80, no. 12, pp. 3618-3622, 1983.

[10] M. Matsuoka and K. T. Jeang, "Human T-cell leukaemia virus type 1 (HTLV-1) infectivity and cellular transformation," Nature Reviews Cancer, vol. 7, no. 4, pp. 270-280, 2007.

[11] T. Igakura, J. C. Stinchcombe, P. K. C. Goon et al., "Spread of HTLV-I between lymphocytes by virus-induced polarization of the cytoskeleton," Science, vol. 299, no. 5613, pp. 1713-1716, 2003.

[12] M.-I. Thoulouze and A. Alcover, "Can viruses form biofilms?" Trends in Microbiology, vol. 19, no. 6, pp. 257-262, 2011.

[13] K. I. Etoh, S. Tamiya, K. Yamaguchi et al., "Persistent clonal proliferation of human T-lymphotropic virus type I- infected cells in vivo," Cancer Research, vol. 57, no. 21, pp. 4862-4867, 1997.

[14] M. Cavrois, I. Leclercq, O. Gout, A. Gessain, S. Wain-Hobson, and E. Wattel, "Persistent oligoclonal expansion of human T-cell leukemia virus type 1 -infected circulating cells in patients with Tropical spastic paraparesis/HTLV-1 associated myelopathy," Oncogene, vol. 17, no. 1, pp. 77-82, 1998.

[15] K. Suemori, H. Fujiwara, T. Ochi et al., "HBZ is an immunogenic protein, but not a target antigen for human Tcell leukemia virus type 1-specific cytotoxic T lymphocytes," Journal of General Virology, vol. 90, no. 8, pp. 1806-1811, 2009.

[16] A. Macnamara, A. Rowan, S. Hilburn et al., "HLA class I binding of HBZ determines outcome in HTLV-1 infection," PLoS Pathogens, vol. 6, no. 9, Article ID e01117, 2010.

[17] C. R. M. Bangham and M. Osame, "Cellular immune response to HTLV-1,” Oncogene, vol. 24, no. 39, pp. 6035-6046, 2005.

[18] Y. Satou, J. I. Yasunaga, M. Yoshida, and M. Matsuoka, "HTLV-I basic leucine zipper factor gene mRNA supports proliferation of adult T cell leukemia cells," Proceedings of the National Academy of Sciences of the United States of America, vol. 103, no. 3, pp. 720-725, 2006.

[19] N. A. Gillet, N. Malani, A. Melamed et al., "The host genomic environment of the provirus determines the abundance of HTLV-1-infected T-cell clones," Blood, vol. 117, no. 11, pp. 3113-3122, 2011.

[20] G. Franchini, R. Fukumoto, and J. R. Fullen, "T-cell control by human T-cell leukemia/lymphoma virus type 1," International Journal of Hematology, vol. 78, no. 4, pp. 280-296, 2003.

[21] N. D. Collins, G. C. Newbound, B. Albrecht, J. L. Beard, L. Ratner, and M. D. Lairmore, "Selective ablation of human Tcell lymphotropic virus type 1 p12(I) reduces viral infectivity in vivo," Blood, vol. 91, no. 12, pp. 4701-4707, 1998.

[22] J. T. Bartoe, B. Albrecht, N. D. Collins et al., "Functional role of pX open reading frame II of human T-lymphotropic virus type 1 in maintenance of viral loads in vivo," Journal of Virology, vol. 74, no. 3, pp. 1094-1100, 2000.

[23] H. Hiraragi, S. J. Kim, A. J. Phipps et al., "Human Tlymphotropic virus type 1 mitochondrion-localizing protein p13 II is required for viral infectivity in vivo," Journal of Virology, vol. 80, no. 7, pp. 3469-3476, 2006.

[24] V. W. Valeri, A. Hryniewicz, V. Andresen et al., "Requirement of the human T-cell leukemia virus p12 and p30 products for infectivity of human dendritic cells and macaques but not rabbits," Blood, vol. 116, no. 19, pp. 3809-3817, 2010.

[25] V. Andresen, C. A. Pise-Masison, U. Sinha-Datta et al., "Suppression of HTLV-1 replication by tax-mediated rerouting of the p13 viral protein to nuclear speckles," Blood, vol. 118, no. 6, pp. 1549-1559, 2011.

[26] Y. Koyanagi, Y. Itoyama, N. Nakamura et al., "In vivo infection of human T-cell leukemia virus type 1 in non-T cells," Virology, vol. 196, no. 1, pp. 25-33, 1993.

[27] J. H. Richardson, A. J. Edwards, J. K. Cruickshank, P. Rudge, and A. G. Dalgleish, "In vivo cellular tropism of human T-cell leukemia virus type 1," Journal of Virology, vol. 64, no. 11, pp. 5682-5687, 1990.

[28] J. I. Yasunaga, T. Sakai, K. Nosaka et al., "Impaired production of naive T lymphocytes in human T-cell leukemia virus type Iinfected individuals: its implications in the immunodeficient state," Blood, vol. 97, no. 10, pp. 3177-3183, 2001.

[29] M. Nejmeddine, V. S. Negi, S. Mukherjee et al., "HTLV-1Tax and ICAM-1 act on T-cell signal pathways to polarize the microtubule-organizing center at the virological synapse," Blood, vol. 114, no. 5, pp. 1016-1025, 2009.

[30] Y. Shimizu, G. A. Van Seventer, K. J. Horgan, and S. Shaw, "Roles of adhesion molecules in T-cell recognition: fundamental similarities between four integrins on resting human T cells (LFA-1, VLA-4, VLA-5, VLA-6) in expression, binding, and costimulation," Immunological Reviews, no. 114, pp. 109-143, 1990.

[31] D. C. Macallan, D. Wallace, Y. Zhang et al., "Rapid turnover of effector-memory CD4+ T cells in healthy humans," Journal of Experimental Medicine, vol. 200, no. 2, pp. 255-260, 2004.

[32] B. Asquith, Y. Zhang, A. J. Mosley et al., "In vivo T lymphocyte dynamics in humans and the impact of human $\mathrm{T}$ lymphotropic virus 1 infection," Proceedings of the National Academy of Sciences of the United States of America, vol. 104, no. 19, pp. 8035-8040, 2007.

[33] Y. Satou, J.-I. Yasunaga, T. Zhao et al., "HTLV-1 bZIP factor induces T-cell lymphoma and systemic inflammation in vivo," PLoS Pathogens, vol. 7, no. 2, Article ID e1001274, 2011.

[34] S. Sakaguchi, T. Yamaguchi, T. Nomura, and M. Ono, "Regulatory T cells and immune tolerance," Cell, vol. 133, no. 5, pp. 775-787, 2008.

[35] Y. Belkaid, "Regulatory $\mathrm{T}$ cells and infection: a dangerous necessity," Nature Reviews Immunology, vol. 7, no. 11, pp. 875888, 2007.

[36] F. Toulza, A. Heaps, Y. Tanaka, G. P. Taylor, and C. R. M. Bangham, "High frequency of CD4+FoxP3+ cells in HTLV1 infection: inverse correlation with HTLV-l-specific CTL response," Blood, vol. 111, no. 10, pp. 5047-5053, 2008.

[37] S. Yamazaki and R. M. Steinman, "Dendritic cells as controllers of antigen-specific Foxp3+ regulatory T cells," Journal of Dermatological Science, vol. 54, no. 2, pp. 69-75, 2009.

[38] S. E. Macatonia, J. K. Cruickshank, P. Rudge, and S. C. Knight, "Dendritic cells from patients with tropical spastic paraparesis are infected with HTLV-1 and stimulate autologous lymphocyte proliferation," AIDS Research and Human Retroviruses, vol. 8, no. 9, pp. 1699-1706, 1992. 
[39] M. Makino, S. Shimokubo, S. I. Wakamatsu, S. Izumo, and M. Baba, "The role of human T-lymphotropic virus type 1 (HTLV-1)-infected dendritic cells in the development of htlv1 - associated myelopathy/tropical spastic paraparesis," Journal of Virology, vol. 73, no. 6, pp. 4575-4581, 1999.

[40] K. S. Jones, C. Petrow-Sadowski, Y. K. Huang, D. C. Bertolette, and F. W. Ruscetti, "Cell-free HTLV-1 infects dendritic cells leading to transmission and transformation of CD4+ T cells," Nature Medicine, vol. 14, no. 4, pp. 429-436, 2008.

[41] M. Vukmanovic-Stejic, Y. Zhang, J. E. Cook et al., "Human CD4+CD25hiFoxp3+ regulatory $\mathrm{T}$ cells are derived by rapid turnover of memory populations in vivo," Journal of Clinical Investigation, vol. 116, no. 9, pp. 2423-2433, 2006.

[42] T. Zhao, Y. Satou, K. Sugata et al., "HTLV-1 bZIP factor enhances TGF- $\beta$ signaling through p300 coactivator," Blood, vol. 118, no. 7, pp. 1865-1876, 2011.

[43] H. Yano, T. Ishida, A. Inagaki et al., "Regulatory T-cell function of adult T-cell leukemia/lymphoma cells," International Journal of Cancer, vol. 120, no. 9, pp. 2052-2057, 2007.

[44] S. Chen, N. Ishii, S. Ine et al., "Regulatory T cell-like activity of Foxp3+ adult T cell leukemia cells," International Immunology, vol. 18, no. 2, pp. 269-277, 2006.

[45] T. Shimauchi, K. Kabashima, and Y. Tokura, "Adult T-cell leukemia/lymphoma cells from blood and skin tumors express cytotoxic T lymphocyte-associated antigen-4 and Foxp3 but lack suppressor activity toward autologous CD8+ T cells," Cancer Science, vol. 99, no. 1, pp. 98-106, 2008.

[46] F. Toulza, K. Nosaka, M. Takiguchi et al., "FoxP3+ regulatory $\mathrm{T}$ cells are distinct from leukemia cells in HTLV-1-associated adult T-cell leukemia," International Journal of Cancer, vol. 125, no. 10, pp. 2375-2382, 2009.

[47] M. Abe, K. Uchihashi, T. Kazuto et al., "Foxp3 expression on normal and leukemic CD4+CD25+ T cells implicated in human T-cell leukemia virus type-1 is inconsistent with Treg cells," European Journal of Haematology, vol. 81, no. 3, pp. 209217, 2008.

[48] K. Karube, K. Ohshima, T. Tsuchiya et al., "Expression of FoxP3, a key molecule in CD4+CD25+ regulatory $\mathrm{T}$ cells, in adult T-cell leukaemia/lymphoma cells," British Journal of Haematology, vol. 126, no. 1, pp. 81-84, 2004.

[49] S. Hori, "Regulatory T cell plasticity: beyond the controversies," Trends in Immunology, vol. 32, no. 7, pp. 295-300, 2011.

[50] D. Larocca, L. A. Chao, M. H. Seto, and T. K. Brunck, "Human T-cell leukemia virus minus strand transcription in infected Tcells," Biochemical and Biophysical Research Communications, vol. 163, no. 2, pp. 1006-1013, 1989.

[51] G. Gaudray, F. Gachon, J. Basbous, M. Biard-Piechaczyk, C. Devaux, and J. M. Mesnard, "The complementary strand of the human T-cell leukemia virus type 1 RNA genome encodes a bZIP transcription factor that down-regulates viral transcription," Journal of Virology, vol. 76, no. 24, pp. 12813 12822, 2002.

[52] T. Usui, K. Yanagihara, K. Tsukasaki et al., "Characteristic expression of HTLV-1 basic zipper factor (HBZ) transcripts in HTLV-1 provirus-positive cells," Retrovirology, vol. 5, article 34, 2008.

[53] M. Saito, T. Matsuzaki, Y. Satou et al., "In vivo expression of the HBZ gene of HTLV-1 correlates with proviral load, inflammatory markers and disease severity in HTLV-1 associated myelopathy/tropical spastic paraparesis (HAM/TSP)," Retrovirology, vol. 6, article 19, 2009.

[54] K. Shimizu-Kohno, Y. Satou, F. Arakawa et al., "Detection of HTLV-1 by means of HBZ gene in situ hybridization in formalin-fixed and paraffin-embedded tissues," Cancer Science, vol. 102, no. 7, pp. 1432-1436, 2011.

[55] M. H. Cavanagh, S. Landry, B. Audet et al., "HTLV-I antisense transcripts initiating in the 3'LTR are alternatively spliced and polyadenylated," Retrovirology, vol. 3, article 15, 2006.

[56] K. Murata, T. Hayashibara, K. Sugahara et al., "A novel alternative splicing isoform of human T-cell leukemia virus type 1 bZIP factor (HBZ-SI) targets distinct subnuclear localization," Journal of Virology, vol. 80, no. 5, pp. 2495-2505, 2006.

[57] F. Rende, I. Cavallari, A. Corradin et al., "Kinetics and intracellular compartmentalization of HTLV-1 gene expression: nuclear retention of HBZ mRNAs," Blood, vol. 117, no. 18, pp. 4855-4859, 2011.

[58] M. Yoshida, Y. Satou, J. I. Yasunaga, J. I. Fujisawa, and M. Matsuoka, "Transcriptional control of spliced and unspliced human T-cell leukemia virus type 1 bZIP factor (HBZ) gene," Journal of Virology, vol. 82, no. 19, pp. 9359-9368, 2008.

[59] K. Doi, X. Wu, Y. Taniguchi et al., "Preferential selection of human T-cell leukemia virus type I provirus integration sites in leukemic versus carrier states," Blood, vol. 106, no. 3, pp. 1048-1053, 2005.

[60] I. Clerc, N. Polakowski, C. Andre-Arpin et al., "An interaction between the human $\mathrm{T}$ cell leukemia virus type 1 basic leucine zipper factor (HBZ) and the KIX domain of p300/CBP contributes to the down-regulation of tax-dependent viral transcription by HBZ," Journal of Biological Chemistry, vol. 283, no. 35, pp. 23903-23913, 2008.

[61] R. Mukai and T. Ohshima, "Dual effects of HTLV-1 bZIP factor in suppression of interferon regulatory factor 1," Biochemical and Biophysical Research Communications, vol. 409, no. 2, pp. 328-332, 2011.

[62] N. Polakowski, H. Gregory, J. M. Mesnard, and I. Lemasson, "Expression of a protein involved in bone resorption, Dkk1, is activated by HTLV-1 bZIP factor through its activation domain," Retrovirology, vol. 7, article 61, 2010.

[63] J. Basbous, C. Arpin, G. Gaudray, M. Piechaczyk, C. Devaux, and J. M. Mesnard, "The HBZ factor of human T-cell leukemia virus type I dimerizes with transcription factors JunB and cJun and modulates their transcriptional activity," Journal of Biological Chemistry, vol. 278, no. 44, pp. 43620-43627, 2003.

[64] S. Thebault, J. Basbous, P. Hivin, C. Devaux, and J. M. Mesnard, "HBZ interacts with JunD and stimulates its transcriptional activity," FEBS Letters, vol. 562, no. 1-3, pp. 165170,2004

[65] I. Lemasson, M. R. Lewis, N. Polakowski et al., "Human TCell leukemia virus type 1 (HTLV-1) bZIP protein interacts with the cellular transcription factor CREB to inhibit HTLV1 transcription," Journal of Virology, vol. 81, no. 4, pp. 1543$1553,2007$.

[66] K. Hagiya, J.-I. Yasunaga, Y. Satou, K. Ohshima, and M. Matsuoka, "ATF3, an HTLV-1 bZip factor binding protein, promotes proliferation of adult T-cell leukemia cells," Retrovirology, vol. 8, article 19, 2011.

[67] T. Ohshima, R. Mukai, N. Nakahara et al., "HTLV-1 basic leucine-zipper factor, HBZ, interacts with MafB and suppresses transcription through a Maf recognition element," Journal of Cellular Biochemistry, vol. 111, no. 1, pp. 187-194, 2010.

[68] T. Zhao, J. I. Yasunaga, Y. Satou et al., "Human T-cell leukemia virus type 1 bZIP factor selectively suppresses the classical pathway of NF- $\kappa$ B," Blood, vol. 113, no. 12, pp. 2755-2764, 2009.

[69] J. Arnold, B. Yamamoto, M. Li et al., "Enhancement of infectivity and persistence in vivo by HBZ, a natural antisense 
coded protein of HTLV-1," Blood, vol. 107, no. 10, pp. 39763982, 2006.

[70] W. J. Grossman, J. T. Kimata, F. H. Wong, M. Zutter, T. J. Ley, and L. Ratner, "Development of leukemia in mice transgenic for the tax gene of human T- cell leukemia virus type I," Proceedings of the National Academy of Sciences of the United States of America, vol. 92, no. 4, pp. 1057-1061, 1995.

[71] M. Yoshida, "Multiple viral strategies of HTLV-1 for dysregulation of cell growth control," Annual Review of Immunology, vol. 19, pp. 475-496, 2001.

[72] C. Z. Giam and K. T. Jeang, "HTLV-1 tax and adult T-cell leukemia," Frontiers in Bioscience, vol. 12, no. 4, pp. 14961507, 2007.

[73] S. J. Marriott and O. J. Semmes, "Impact of HTLV-I Tax on cell cycle progression and the cellular DNA damage repair response," Oncogene, vol. 24, no. 39, pp. 5986-5995, 2005.

[74] H. Hasegawa, H. Sawa, M. J. Lewis et al., "Thymus-derived leukemia-lymphoma in mice transgenic for the Tax gene of human T-lymphotropic virus type I," Nature Medicine, vol. 12, no. 4, pp. 466-472, 2006.

[75] Y. Iwakura, M. Tosu, E. Yoshida et al., "Induction of inflammatory arthropathy resembling rheumatoid arthritis in mice transgenic for HTLV-I," Science, vol. 253, no. 5023, pp. 10261028, 1991.

[76] T. Ohsugi, T. Kumasaka, S. Okada, and T. Urano, "The Tax protein of HTLV-1 promotes oncogenesis in not only immature T cells but also mature T cells," Nature Medicine, vol. 13, no. 5, pp. 527-528, 2007.

[77] H. Zhi, L. Yang, Y.-L. Kuo, Y.-K. Ho, H.-M. Shih, and C.Z. Giam, "NF- $\kappa$ B hyper-activation by HTLV-1 tax induces cellular senescence, but can be alleviated by the viral antisense protein HBZ," PLoS Pathogens, vol. 7, no. 4, Article ID e1002025, 2011.

[78] S. Hino, S. Katamine, K. Kawase et al., "Intervention of maternal transmission of HTLV-I in Nagasaki, Japan," Leukemia, vol. 8, no. 1, pp. S68-S70, 1994.

[79] A. Bazarbachi, Y. Plumelle, J. Carlos Ramos et al., "Metaanalysis on the use of zidovudine and interferon-alfa in adult T-cell leukemia/lymphoma showing improved survival in the leukemic subtypes," Journal of Clinical Oncology, vol. 28, no. 27, pp. 4177-4183, 2010.

[80] A. Utsunomiya, Y. Miyazaki, Y. Takatsuka et al., "Improved outcome of adult $\mathrm{T}$ cell leukemia/lymphoma with allogeneic hematopoietic stem cell transplantation," Bone Marrow Transplantation, vol. 27, no. 1, pp. 15-20, 2001.

[81] J. Okamura, A. Utsunomiya, R. Tanosaki et al., "Allogeneic stem-cell transplantation with reduced conditioning intensity as a novel immunotherapy and antiviral therapy for adult Tcell leukemia/lymphoma," Blood, vol. 105, no. 10, pp. 41434145, 2005.

[82] M. Kannagi, T. Ohashi, N. Harashima, S. Hanabuchi, and A. Hasegawa, "Immunological risks of adult T-cell leukemia at primary HTLV-I infection," Trends in Microbiology, vol. 12, no. 7, pp. 346-352, 2004.

[83] I. Lemasson, N. J. Polakowski, P. J. Laybourn, and J. K. Nyborg, "Transcription regulatory complexes bind the human T-cell leukemia virus $5^{\prime}$ and $3^{\prime}$ long terminal repeats to control gene expression," Molecular and Cellular Biology, vol. 24, no. 14, pp. 6117-6126, 2004.

[84] J. K. Nyborg, D. Egan, and N. Sharma, "The HTLV-1 Tax protein: revealing mechanisms of transcriptional activation through histone acetylation and nucleosome disassembly," Biochimica et Biophysica Acta, vol. 1799, no. 3-4, pp. 266-274, 2010.
[85] Y. Taniguchi, K. Nosaka, J. I. Yasunaga et al., "Silencing of human T-cell leukemia virus type I gene transcription by epigenetic mechanisms," Retrovirology, vol. 2, article 64, 2005.

[86] B. Zimmerman, A. Sargeant, K. Landes, S. A. Fernandez, C.-S. Chen, and M. D. Lairmore, "Efficacy of novel histone deacetylase inhibitor, AR42, in a mouse model of, human Tlymphotropic virus type 1 adult T cell lymphoma," Leukemia Research, vol. 35, no. 11, pp. 1491-1497, 2011.

[87] A. Lezin, N. Gillet, S. Olindo et al., "Histone deacetylasemediated transcriptional activation reduces proviral loads in HTLV-1-associated myelopathy/tropical spastic paraparesis patients," Blood, vol. 110, no. 10, pp. 3722-3728, 2007.

[88] G. Belrose, A. Gross, S. Olindo et al., "Effects of valproate on Tax and HBZ expression in HTLV-1 and HAM/TSPT lymphocytes," Blood, vol. 118, no. 9, pp. 2483-2491, 2011.

[89] P. V. Afonso, M. Mekaouche, F. Mortreux et al., "Highly active antiretroviral treatment against STLV-1 infection combining reverse transcriptase and HDAC inhibitors," Blood, vol. 116, no. 19 , pp. 3802-3808, 2010. 


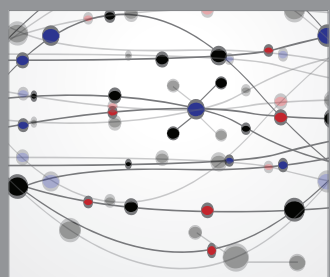

The Scientific World Journal
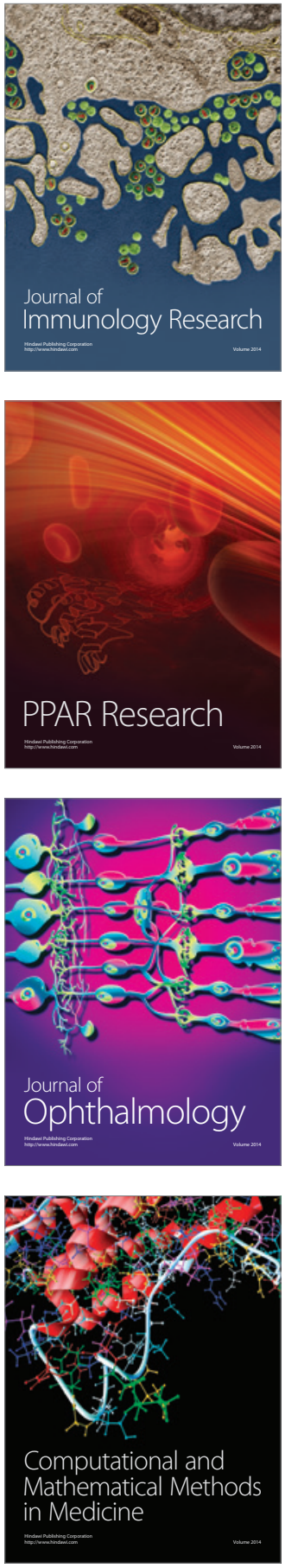

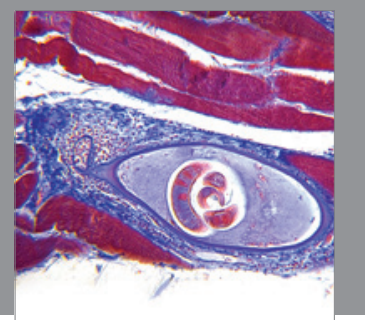

Gastroenterology

Research and Practice
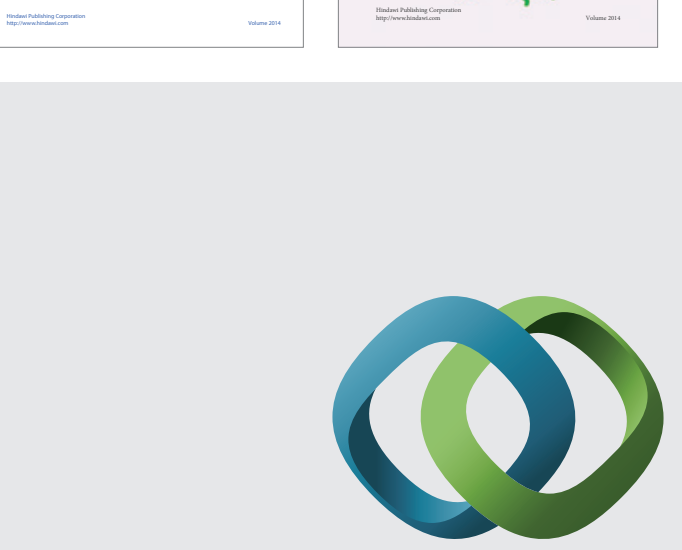

\section{Hindawi}

Submit your manuscripts at

http://www.hindawi.com
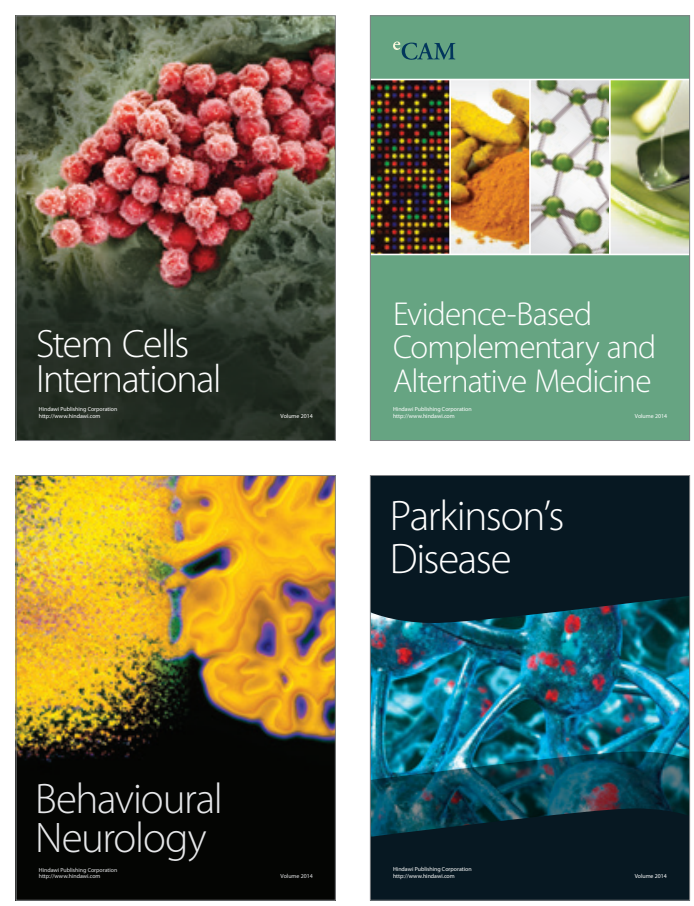

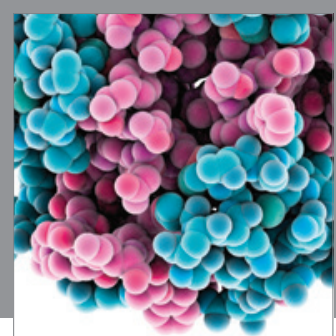

Journal of
Diabetes Research

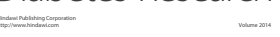

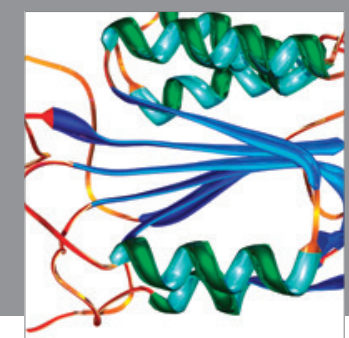

Disease Markers
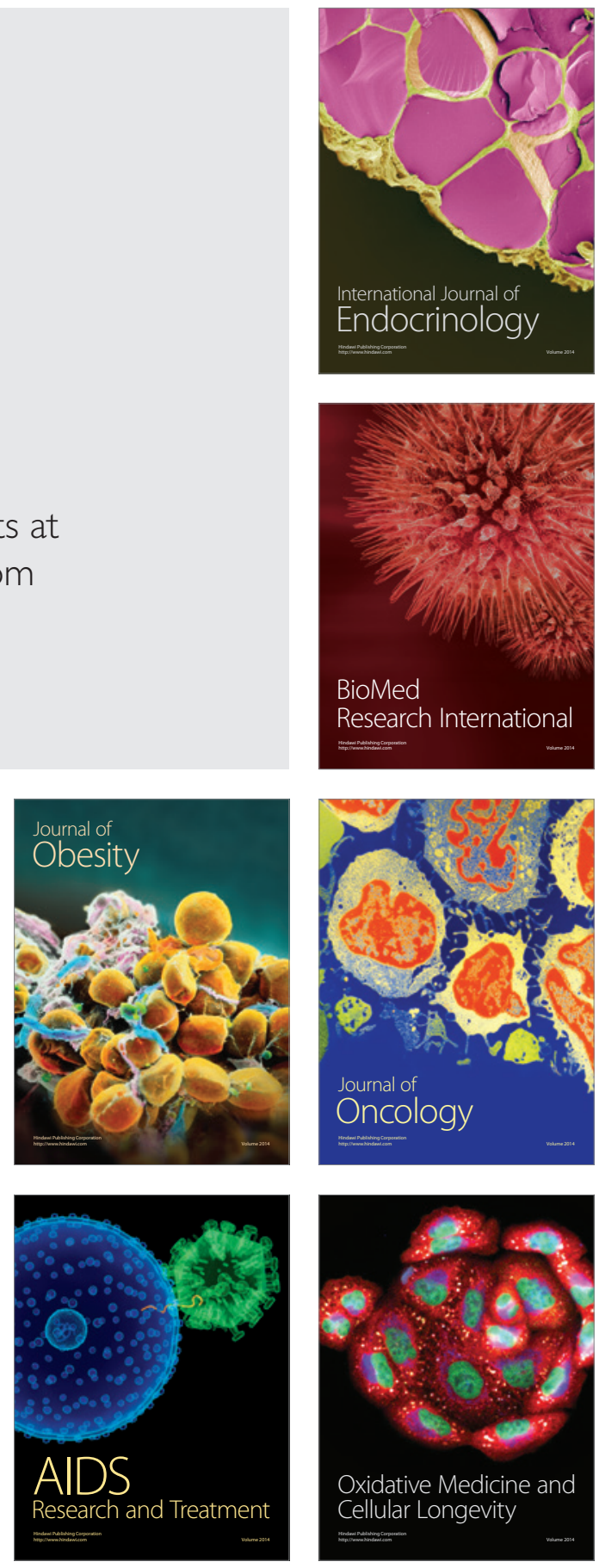